\title{
Nerve sheath tumor surgery: case-guided discussion of ambiguous findings, appropriateness of removal, repeated surgery, and nerve repairs
}

\author{
Thomas Kretschmer, M.D., Ph.D., ${ }^{1}$ Gregor Antoniadis, M.D., Ph.D., ${ }^{1}$ \\ Christian Heinen, M.D., ${ }^{1}$ Wolfgang Börm, M.D., Ph.D., ${ }^{2}$ Christian Scheller, M.D., ${ }^{1}$ \\ Hans-Peter Richter, M.D., Ph.D., ${ }^{1}$ ANd Ralph W. Koenig, M.D. ${ }^{1}$ \\ ${ }^{1}$ Neurosurgical Department, Universität Ulm; and ${ }^{2}$ Neurosurgical Department, \\ Diakonissenanstalt Flensburg, Germany
}

\begin{abstract}
$\checkmark$ In this article the authors attempt to raise awareness of the pitfalls and controversial issues in nerve tumor surgery. In a case-guided format, examples of ambiguous findings, inappropriate tumor removal, repeated surgery, and nerve repairs are provided. The authors also discuss the need to establish a correct diagnosis preoperatively and to avoid the erroneous identification of malignant peripheral nerve sheath tumors (MPNSTs). They emphasize that not all of the principles of soft tissue sarcoma treatment protocols are applicable to MPNST. A situation of repeated surgery for supposedly malignant tumor is described, and an outline of the indications for, and an approach to, repair after lesion removal is given.
\end{abstract}

KEY Words - imaging - microsurgery - nerve repair • peripheral nerve sheath tumor • repeated surgery $\bullet$ soft tissue sarcoma

I

$\mathrm{N}$ this article, we attempted to detail a few of the controversial but inherent issues in nerve tumor surgery. We intended to focus on some of the somewhat infrequently covered, but nevertheless practically important, aspects such as inappropriate tumor removal, unexpected findings, necessary repeated surgery, and grafting after tumor excision.

What is an inappropriate tumor removal? The question might be easy to answer with regard to benign tumors like schwannomas and neurofibromas: In the era of microsurgery a new major functional deficit after surgery is usually unacceptable. It has been shown in recent series that these tumors can be enucleated and resected with very favorable functional results. ${ }^{11,18,27}$ The question is more difficult to answer with regard to MPNSTs. Appropriate or inappropriate removal can in either case leave the patient with a functional deficit. With this pathological entity, inappropriate would imply leaving the tumor, thus exposing the

Abbreviations used in this paper: BPNST = benign peripheral nerve sheath tumor; $\mathrm{CT}=$ computed tomography; FDG-PET = fluorine-18-labeled fluorodeoxyglucose-positron emission tomography; LSUMC = Louisiana State University Medical Center; MPNST = malignant PNST; MR = magnetic resonance. patient to a greater risk of recurrence and spread. Therefore, repeated surgery would be the logical response. At this stage, however, the controversy begins. What form of resection is most appropriate: tumor-free stump margins, en bloc resection, or limb ablation?

Sometimes we face the opposite scenario: too much tumor has been resected. Worse than merely inappropriate is probably the resection of a benign tumor together with the parent nerve. Such an event can be inadvertent due to misconception of the underlying pathophysiology and anatomy, failure to respect microsurgical principles, or simply an incorrect initial pathology report documenting malignant features that, in fact, did not exist.

In brief, we attempted to raise awareness of some controversial issues and pitfalls of nerve tumor surgery. This report is by no means intended as a complete account. Cases featured will demonstrate the difficulty in identifying a malignant nerve tumor preoperatively, provide opportunities to discuss when soft tissue sarcoma surgery principles may be inappropriate, and offer two examples of repeated surgery, one for a supposedly malignant tumor. We also outline our indications for repair after tumor removal.

Apart from review, we chose exemplary cases from a 
series of 169 PNSTs (excluding true sarcomas of mesenchymal origin) surgically treated between January 1990 and April 2006 to illustrate, underline, and discuss some points believed to be relevant to our topic. Consequently and intentionally, this paper does not have a traditional format. The critical points of the cases presented will be discussed individually with each case.

\section{Appropriateness of Tumor Removal}

With the 1994 publication of Donner and colleagues"11 data, it became evident that lesions could be removed with unchanged or improved motor function in $87 \%$ of 76 patients with schwannomas and in $90 \%$ of 99 patients with neurofibromas. An old dogma that neurofibromas can only be resected by sacrificing the parent nerve was refuted. By strictly applying microsurgical principles and interfascicular dissection techniques, these authors demonstrated postoperative improvement or resolution of pain syndromes in $88 \%$ of 99 monitored patients. A 2005 update with 397 tumors is the largest series to date. ${ }^{27}$ It included 28 MPNSTs, and the authors concluded that despite aggressive limb-ablation or limb-sparing surgery plus adjunctive therapy, these lesions remain associated with high morbidity and mortality rates. Which principles should apply in the resection of MPNSTs: should one perform surgical removal with tumor-free margins or en bloc resection (wide resection) with limb sparing, or does the extremity have to be ablated to improve the chances for years of survival? Based on the results of a 1998 study, Angelov and associates ${ }^{1,2}$ proposed an algorithm detailing wide resection and adjunctive radiotherapy. Of 18 MPNSTs, 16 involved localized disease; in 11 of these 16 cases, tumor-free surgical margins were achieved. Patients in these 18 cases remained disease free during a mean follow-up of 33.7 months. Notably, it was easier to obtain tumor-free margins with lesions smaller than $5 \mathrm{~cm}$. Despite lesion size, however, a high tumor grade remained a significant negative prognostic factor. Tumor grades assigned following en bloc resection were higher than those based on the initial biopsy. The amount of tissue sent for pathological study is decisively important when identifying an MPNST, and even more so for grading. (We later outline the details of a case [Case 4] in which this factor played an important role.) Obviously key is maintaining tumor-free margins. Which of the stated procedures is the most appropriate for a given situation? These questions remain to be conclusively answered. Individual case numbers are still too small to draw general conclusions. In view of the low 0.001 incidence of MPNSTs in the general population, ${ }^{3,12}$ it is quite obvious why it is so difficult to gather randomized, multicenter data to improve the level of evidence. Nevertheless, many nerve surgeons assert that there are probably subgroups of these tumors that do not behave as aggressively as others, especially the ones more distal in the limb and with a lower grade as well as some sporadic ones unrelated to neurofibromatosis..$^{5,1939,48}$ Of course, every tumor surgeon would prefer to be only as aggressive as necessary and to tailor the approach according to the grade and anticipated behavior of the neoplasm. Unfortunately, there are not enough data beyond those offered by case reports to guide us in that regard.

What are the current principles? This issue is a lengthy discussion on its own, and we refer the reader to articles de- voted to this matter. Nevertheless, the crucial point is that with regard to treatment, MPNSTs are listed in the World Health Organization classification of diseases under soft tissue sarcomas and are said to account for 5 to $10 \%$ of these lesions.,12 Given that soft tissue sarcomas have a mesodermal origin and that PNSTs have a neuroectodermal origin, there is some concern that the World Heath Organization category is incorrect. The crucial point is that treatment algorithms are well established for soft tissue sarcomas. (For an example of a current multicenter study, which is slated to include 75 cases, see the clinical trials website of the National Cancer Institue: http://www.cancer.gov./clinicaltrials/ NCI-06-C-0043\#Contactinfo_CDR0000465364.) In turn, the existence of such algorithms implies that nerve surgeons can apply the same principles in MPNST treatment. The goal in soft tissue sarcoma treatment is to establish diagnosis before surgery (the following website links to an algorithm for the interdisciplinary treatment of soft tissue sarcoma at the University of Ulm, Germany: www.tumorzentrumulm.de). In soft tissue sarcoma, the cutting-needle biopsy is the main choice among biopsy procedures because of its quoted 93\% accuracy rate, ${ }^{13}$ with incisional biopsy mentioned as an alternative. Note, however, that nerve surgeons and neuropathologists oppose needle biopsy procedures for nerves because the risk of potential harm (pain and functional deficit) is far too high and the likelihood of obtaining a conclusive diagnosis is far too low with the small samples. Biopsy samples of benign nerve tumors can also be obtained, but there is the potential of damaging intact nerves with the procedure. Therefore, for a potential MPNST the only choice is incisional biopsy, which in turn means nerve exploration.

If the diagnosis of a soft tissue sarcoma is confirmed, staging is the next step, followed by an interdisciplinary consultation. Recommended staging modalities include MR imaging if the extremities and head and neck are involved, ${ }^{20}$ abdominal CT if the lesion is retroperitoneal, ${ }^{21}$ chest CT if the tumor is larger than $5 \mathrm{~cm},{ }^{17}$ and abdominal CT if a liposarcoma has been diagnosed (because they metastasize to the retroperitoneal space and the liver). ${ }^{8}$ Staging via FDGPET apparently has a high sensitivity for soft tissue sarco$\mathrm{ma}^{35}$ and could be useful for follow-up. Its discriminative power to differentiate G1/G2 osteosarcoma from benign tumors is supposedly weak. ${ }^{41}$ The role of skeletal scintigraphy seems to be confined to myxoid liposarcoma. Staging is followed by excision, with wide resection the goal, which would imply en bloc resection with $1-\mathrm{cm}$ resection margins (R0). The "tumor should not be seen."'14 If only $0.5-\mathrm{cm}$ resection margins can be achieved, adjuvant radiotherapy is proposed. ${ }^{40} \mathrm{~A}$ role for neoadjuvant radiotherapy is acknowledged if there is no possibility for a primary $\mathrm{R} 0$ intervention. ${ }^{7,10,36,40}$ In cases of a microscopic tumor rest (R1), macroscopic tumor rest (R2), or insufficiently large safety margin (marginal resection), repeated resection is recommended. ${ }^{24}$ In the event that an MPNST on a brachial plexus element is resected with tumor-free margins, it qualifies as an instance of marginal resection only; therefore, according to the soft tissue sarcoma protocol, this case would require repeated resection. Does this case mean that it is appropriate to repeat the resection together with sacrifice of all the vessels and the entire plexus, which basically means a shoulder-limb amputation? We are unable to answer this question and will not attempt to. Decisions in this regard 
will always be made on a very individual basis. Nevertheless, we believe it is important to draw attention to, and raise awareness about, controversial points such as these.

The prognosis of soft tissue sarcomas is highly influenced by disease stage, tumor grade, ${ }^{9,47}$ patient age, and lesion size, depth, location (limbs, trunk, or head/neck), and histological features, with MPNST being in the worst category if listed under sarcomas. A nomogram to predict the postoperative 12-year sarcoma-specific death rate is available. ${ }^{25}$

\section{Ambiguous Findings}

If an unclear painless or painful mass is noted, a prompt workup with MR imaging should be performed. Most PNSTs are moderatly inhomogeneous on MR images, with intermediate to bright signal on T1-weighted images. To date, there is no imaging method that can differentiate a schwannoma from a neurofibroma or reliably distinguish an MPNST from a BPNST. On the T2- and proton-weighted sequences, there is a higher signal, at times with some inhomogeneity for a malignant lesion. ${ }^{44}$ Features suggesting a PNST on CT and MR images include a fusiform shape, an entering and exiting peripheral nerve, and sometimes associated muscle atrophy. With a BPNST, a fascicular and split-fat sign have been described on T2-weighted images. These terms refer to a ringlike, surrounding fat signal and a concentric underlying hypointensity referring to fascia on the axial T2 view. ${ }^{34}$ A so-called target sign is apparently more abundant in BPNSTs; that is, a target pattern on axial views with a peripheral hyperintense rim and central low intensity. These changes are believed to correspond histologically to an outer zone of peripheral myxomatous tissue and a central zone of fibrocollagenous tissue. Even the initial description by Varma et al. ${ }^{45}$ identified it in only $12(52 \%)$ of 23 BPNSTs., ${ }^{43,37,38}$ Inhomogeneity in MPNSTs seems to reflect coexisting areas of hypo- and hypercellularity rather than regressive changes like necrosis or hemorrhage. ${ }^{33,44}$ Initial efforts to discern neurofibromas and plexiform neurofibromas from MPNSTs have been successful recently with the use of FDG-PET, ${ }^{6,15,16,43}$ However, false-positive results have been detected as well. ${ }^{22}$ Unlike in soft tissue sarcomas, ultrasonography does not really help to differentiate between BPNSTs and MPNSTs. A tumor is apparently more likely to be benign if the lesion is echorich, not excessively vascularized, and mobile to palpation.$^{42}$ In most cases, the suspicion of potentially facing malignancy arises from clinical clues: morphological features such as a size larger than $5 \mathrm{~cm}$ and firmness, and symptoms such as neurological deficit, pain (especially resting pain), and a relatively rapid progression of these signs are highly suspicious for malignancy. If a PNST is superficial enough, then it can be palpated as a relatively firm swelling. Usually a Hoffmann-Tinel sign can be elicited on tapping the mass. The electrical dysesthesias are distributed along the course of the nerve. A classic finding with palpation of the more benign tumors is mobility perpendicular to the course of the nerve, with immobility along the long axis. Malignant PNSTs tend to be very firm, more painful, and not as mobile, given that they are more fixed to surrounding tissue. However, there are always cases in which these features will not be displayed in a straightforward, clear-cut pattern.

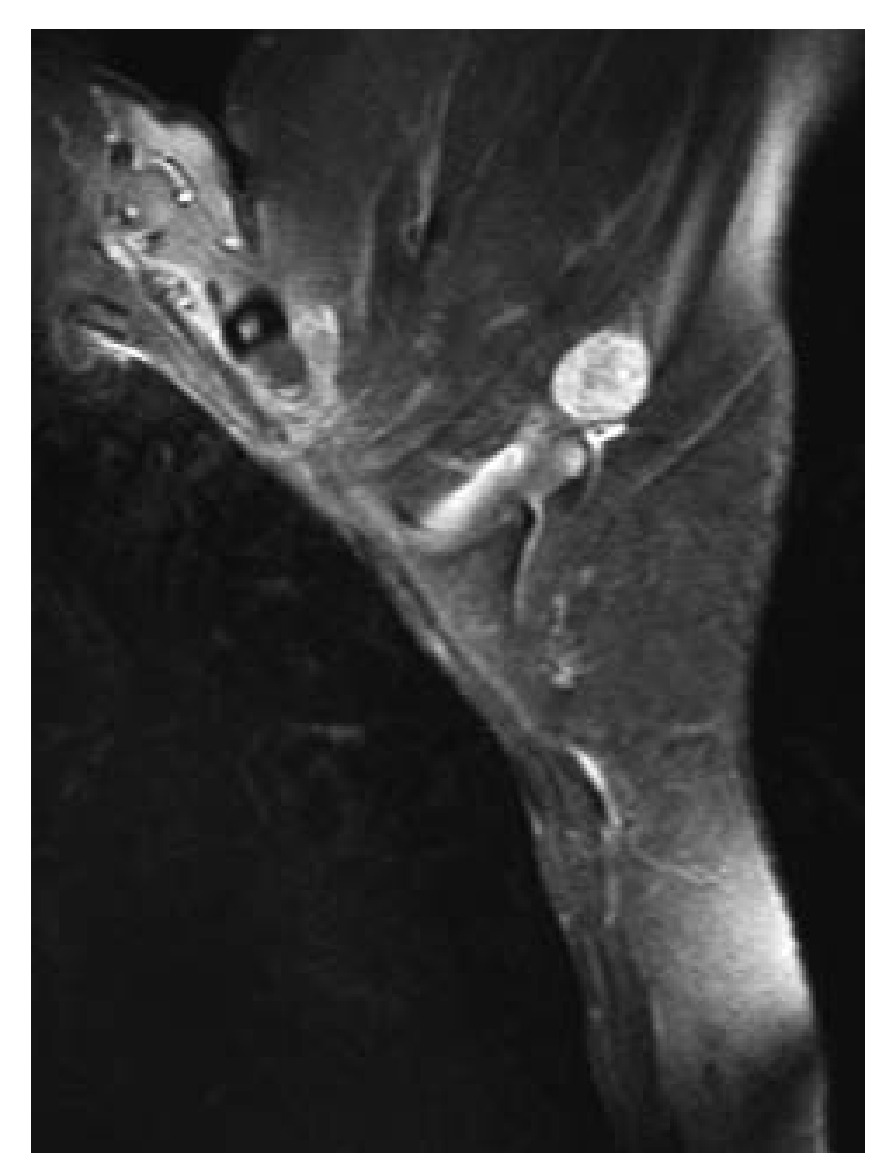

FIG. 1. Case 1. Left axilla MR image obtained in a 49-yearold woman harboring a carcinoma in situ, showing a well-circumscribed nodular lesion with a bright signal. Intraoperatively, a BPNST from the axillary nerve was confirmed.

\section{Case 1}

Examination. This 49-year-old woman harbored a left in situ breast carcinoma (Fig. 1).

Operation. She underwent excision via a small transaxillary-retropectoral approach, with a result that was very pleasing cosmetically.

Postoperative Course. On follow-up 2 weeks after surgery, she reported the onset of local left axillary pain/discomfort and some hypersensitivity around the left shoulder cap. Deep axillary palpation demonstrated good mobility of a firm and slightly tender lesion. There was no motor deficit. An MR imaging workup revealed a 2-cm round lesion in the axilla and the distal infraclavicular brachial plexus, at a cord to nerve level. The patient's clinical presentation suggested a soft tissue tumor arising from the axillary nerve. Metastasis - for example, a positive lymph node - certainly would have to be included in the differential diagnosis. A nerve could be seen entering and exiting the lesion. There was no target sign, and the core of the lesion was hypointense.

Second Operation. A decision was made to explore via a transaxillary incision with potential extension to the previ- 
ous axillary-lateral-retropectoral incision. Should we be in doubt about the pathophysiology, an excisional biopsy procedure was planned instead of tumor removal. Intraoperatively, it became evident that the lesion was a PNST arising from the axillary nerve. Under microscopic magnification, clear planes between the fascicles and tumor mass could be seen; therefore, straightforward enucleation was performed together with resection of the inleading and outleading fascicle $3 \mathrm{~mm}$ past the tumor. At no point during the operation was there doubt about the benign nature of the lesion. Stimulation before and during tumor removal guided the dissection, and good responses were obtained after enucleation.

The patient's history and imaging features might well have matched those for a malignant metastatic breast lesion.

\section{Case 2}

History and Examination. This 72-year-old man noticed painless numbness to the dorsal aspect of his left upper arm, hand, and thumb, which had acutely developed overnight. He had a medical history of radiotherapy and chemotherapy for non-Hodgkin lymphoma 17 years earlier. On presentation, his wrist and finger extensors had an LSUMC ${ }^{30}$ Grade of 0 as was his latissimus dorsi. He harbored a lesion in the left infraclavicular, subaxillary space. On deep axillary palpation, firmness and mobility could not be properly assessed given the depth of the lesion. No Hoffmann-Tinel sign could be elicited; his triceps muscle was very sensitive to palpation. On MR imaging (Fig. 1), there was a well-circumscribed, round lesion with a concentric enhancing ring and central hypointensity with inhomogeneity. There were no signs of infiltration into surrounding tissue. Applying the aforementioned criteria, the MR image might well have represented a BPNST: apart from the clear margins of the round/oval lesion, the signal reaction on axial views could be interpreted as a target sign. Note also that there was some inhomogeneity. A decision was made to explore the lesion after staging was negative. Prior to admission for surgery, progressive pain developed in his left shoulder and axilla. The preoperative differential diagnoses included MPNST, metastasis, recurrency of the non-Hodgkin lymphoma, and PNST.

Operation. The infraclavicular plexal elements could be dissected in the usual manner; there were no adhesions or signs suggestive of invasion. After medialization of the lateral cord and identification of the axillary artery, the deeper plane of the posterior cord was entered. At the level of the trifurcation into the radial, axial, and thoracodorsal nerve, a 4- to 5-cm mass emanating from the thoracodorsal nerve was identified, which compressed but had not infiltrated the radial nerve. The axillary nerve was unaffected, and on stimulation it evoked a good response from the deltoid muscle. On microscopic viewing and attempted dissection of the thoracodorsal nerve, it became clear that it was impossible to discern and enter a plane between the fascicles and tumor. Additional features suggesting malignancy intraoperatively included adherence to surrounding tissue, lack of proper perpendicular mobility, and firmness. A decision was made to resect the tumor together with the nerve.

Postoperative Course. Histopathological study results confirmed an MPNST, with a regional MIB-1 proliferation in-

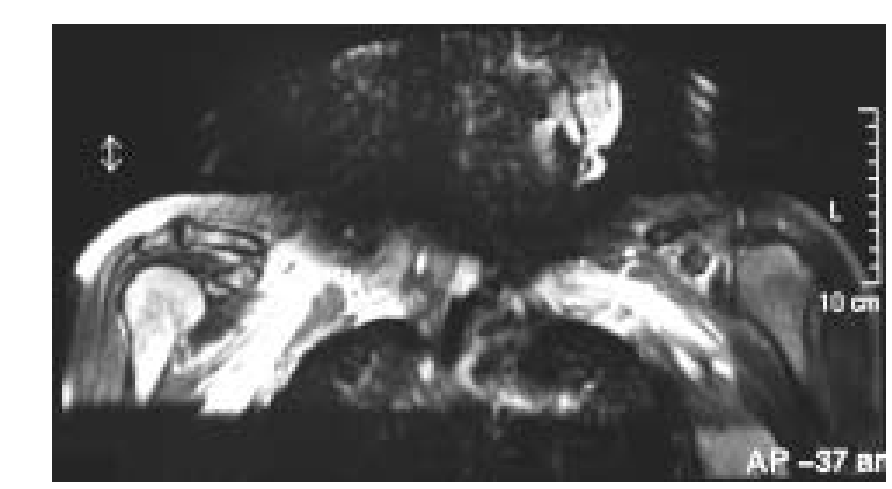

FIG. 2. Case 2. Upper thorax MR image obtained in a 72-yearold patient, demonstrating a well-circumscribed round lesion with a central hypointense signal and a marginal bright signal. The imaging features could suggest a target sign; however, the diagnosed PNST on the thoracodorsal nerve turned out to be highly malignant.

dex of up to $20 \%$; there were up to 15 mitoses/hpf. The options were discussed with the patient, and he decided against repeated resection; adjuvant therapy was not an option. The first signs of local recurrence were apparent on MR imaging 3 months thereafter. Motor deficits progressed rapidly, initially involving the rest of the posterior cord-supplied musculature and then that supplied by the lateral and medial cord. A pain syndrome developed, requiring palliative management. The patient succumbed to the disease 14 months postoperatively.

Note that Ducatman et al. ${ }^{12}$ have described the appearance of MPNST at previously irradiated sites, with a latency of 16.9 years; a coincidence perhaps, but nevertheless striking.

\section{Imaging With Malignant Features}

Case 3

History and Examination. This 17-year-old asymptomatic boy with known neurofibromatosis presented with a massive $17 \times 8-\mathrm{cm}$ left retroperitoneal mass (Fig. 3). The lesion became evident during a routine follow-up and apparently had not been diagnosed 9 months previously, although outside hospital films were unobtainable. The now recognizable, painless, left retroperitoneal mass had displaced the left kidney considerably upward and obstructed its outflow. There was no pain, however, and the patient was otherwise healthy and fit. On MR imaging, the lesion showed clear margins with no signs of invasion into surrounding tissue but did have a very inhomogeneous internal signal with mixed areas of very marked hypo- and hyperintensity. The lesion's size, internal signal structure, association with neurofibromatosis, and supposedly fast growth were interpreted as potentially malignant features. This patient's case was the subject of the hospital's interdisciplinary tumor board conference, and preoperative planning included staging in expectation of a potential malignancy.

Operation. The tumor was resected via a transperitoneal approach (Fig. 4). The lesion was a highly cellular but benign schwannoma. Pathological features included focal cysts without necrosis, spindle-shaped tumor cells, no sig- 

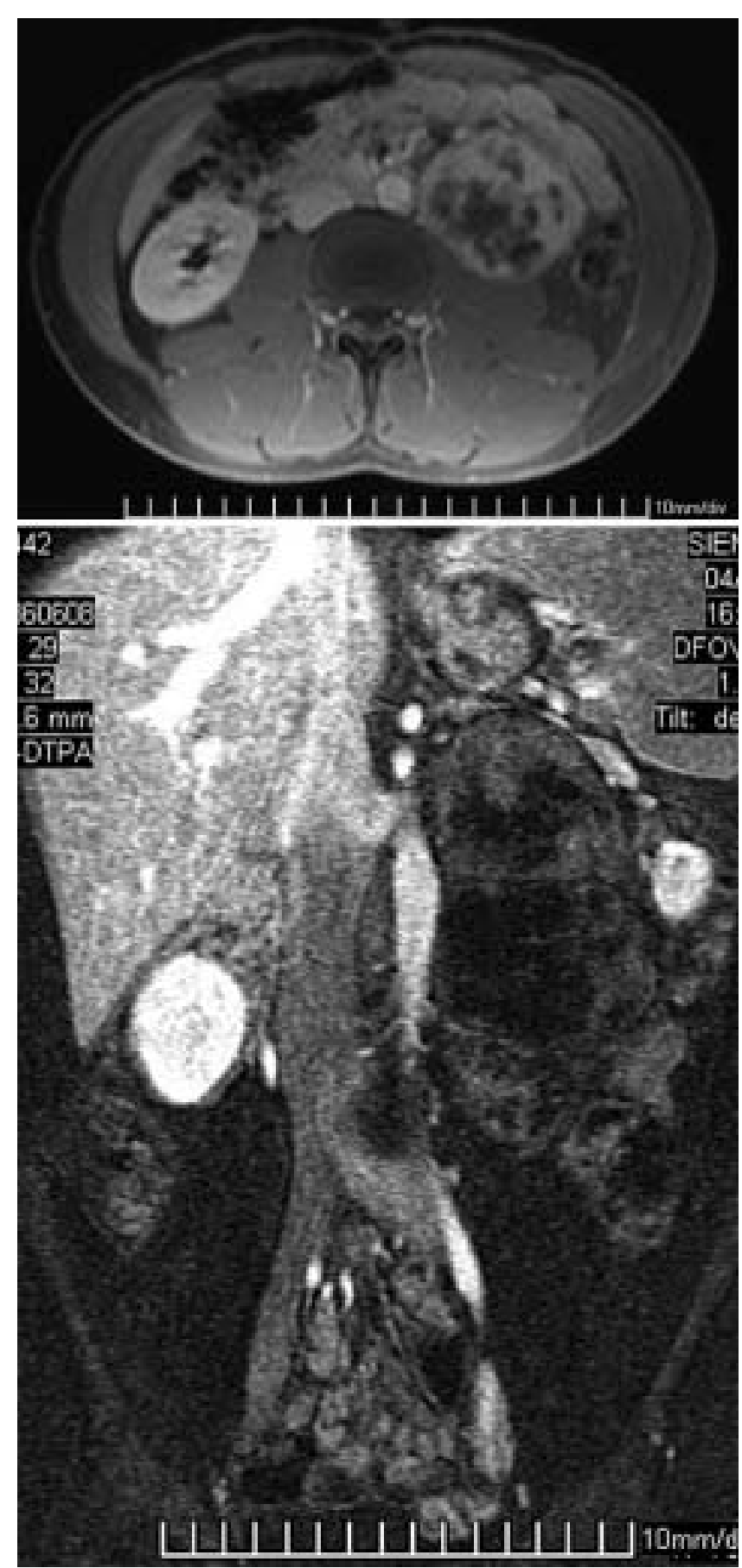

FIG. 3. Upper: Axial MR image obtained in a 17-year-old patient with neurofibromatosis, in whom malignant features were discovered, showing a round circumscribed mass within the left retroperitoneal space. Lower: Coronal MR image, anteroposterior view, demonstrating a very inhomogeneous internal signal with marked areas of hypo- and hyperintensity. The kidney on the left is dislocated.

nificant atypia, cells aligned in a school-of-fish pattern, only marginal palisading of nucleoli, focal areas of hemosiderin pigment, and only rare mitoses ( 1 mitosis per 25 hpf). Immunohistochemical analysis demonstrated strong S100 protein expression, and MIB-1 positivity was a max-

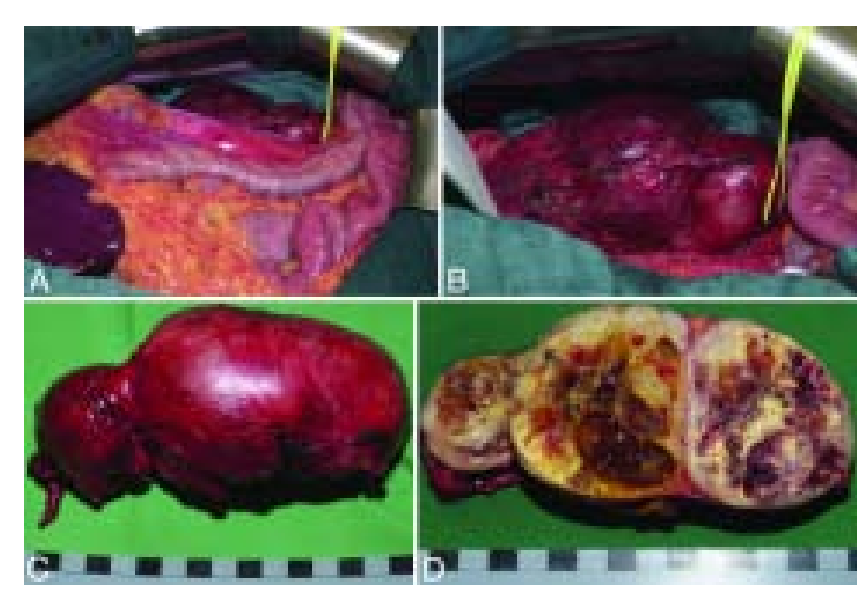

FIG. 4. Case 3. Photographs depicting stages during resection of a left-sided retroperitoneal PNST. A: The transperitoneal approach was used. B: The tumor is delivered and resected from small inleading and outleading nerve branches off the paravertebral plexus. C: Macroscopic view of the $17 \times 8-\mathrm{cm}$ lesion. D: Longitudinal section demonstrating the regressive changes with cystic and hemorrhagic areas. Pathological studies confirmed a benign, hypercellular schwannoma.

imum of 5\% of tumor cells. The cellular schwannoma was described by Woodruff et al. ${ }^{46}$ in 1981 as a variant, which is "sometimes mistaken for a malignant tumor."

In summary, it seems important not to rely totally on supposedly clear imaging or conclusive clinical textbook features. It is crucial to be prepared for potential different surgical strategies dependent on the intraoperative findings. One must design several treatment plans preoperatively. It is advisable to discuss all possible scenarios with the patient before surgery, including malignancy even when it might not readily be expected. Relatedly, discussions should occur to prevent radicality in a benign situation.

\section{Repeated Surgery: Assumed Malignancy}

At times the peripheral nerve surgeon might be confronted with incomplete tumor removal of a BPNST or MPNST. These cases certainly are not the easiest to treat.

\section{Case 4}

History and Examination. This 66-year-old man underwent surgery elsewhere for a presumed lipoma in his left ulnar side forearm. He presented to us with a firm, painless, mobile swelling without sensorimotor deficits. He also exhibited minimal abduction weakness in his left little finger, no sensory deficit, and a positive Hoffmann-Tinel sign with radiation to the left little finger. A preoperative MR image showed a 4-cm left medial forearm mass supposedly emanating from the left ulnar nerve. There was distinct contrast enhancement and an irregular margin, but none of the typical fusiform appearance of a schwannoma. At the ulnar side aspect there was no clear margin, and features were more in keeping with potential infiltration of the mass into soft tissue. The options were discussed with the patient. In the case of doubt regarding the nature of the lesion, the procedure was to be staged again until a final diagnosis could be reached. In the case of a malignancy, the patient preferred resection with tumor-free margins. 


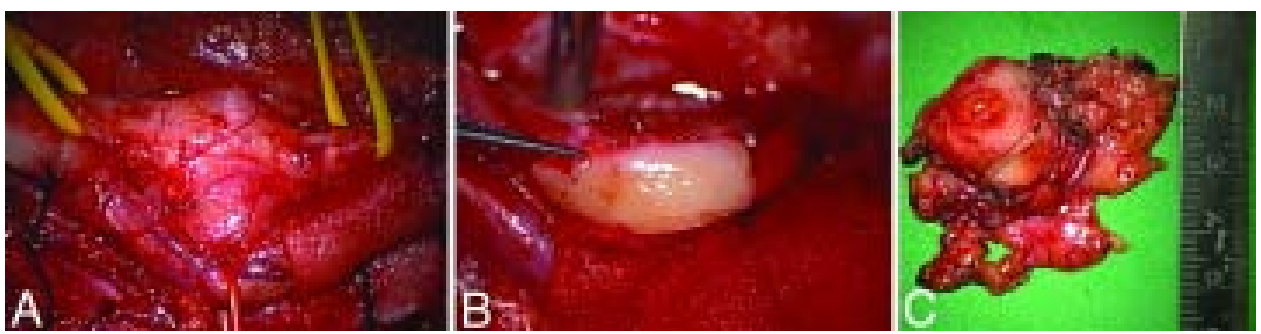

FIG. 5. Case 4. Photographs obtained during surgery to remove a PNST. A: Repeated surgery was undertaken to remove a supposed MPNST, according to a biopsy. The tumor is arising from the left midforearm ulnar nerve. On the ulnar side of the mass, the tumor seems to infiltrate into the soft tissue. B: Incision on the exophytic side shows a yellow-white shiny surface, features in keeping with a schwannoma. Results of pathological studies confirmed a schwannoma; there were no malignant features. Only the infiltrated area showed scarring. C: The tumor in toto, together with excised soft tissue.

Operation. At the first operation elsewhere, a biopsy specimen was taken from the tumor after it became apparent that the presumed lipoma was a PNST. The pathology report documented a low-grade MPNST as opposed to a cellular schwannoma; however, a note was made that the biopsy specimen was too small for a conclusive decision. Figure $5 \mathrm{~A}$ shows the medial aspect of the tumor after the second (our) exposure. The exophytic appearance is evident. The tumor seems to have invaded surrounding tissue, although it is impossible to macroscopically differentiate invasion from scarring due to previous surgery. Circumferential inspection in combination with excision of a sizeable wedge of the exophytic (nonfascicular) tumor portion for quick section gave decisive clues. With magnification and illumination, it was evident that the main caliber of the ulnar nerve had been dislocated to the radial side and that there were healthy fascicles and a clear plane between them and a pseudo-tumor capsule. Quick section gave no indication of malignant features. A benign tumor with scar tissue due to prior operation was thought to be more likely, and the tumor was enucleated from the nonnervous, ulnar side. Dissection from the distal and proximal virgin nerve tissue enabled one to develop planes between tumor and fascicles. The tumor was excised completely with some surrounding soft tissue, without creating new neurological deficits. A definitive pathology report confirmed benign schwannoma and scar tissue on the ulnar side. Figure 5B and $\mathrm{C}$ shows the typical yellow-white surface of the schwannoma and the tumor in toto.

Thus, in this case it was rather difficult to distinguish between soft tissue scarring induced by prior surgery and a malignant nerve tumor mass. This case also illustrates how difficult it can be to make a definite diagnosis from small samples. This case also makes one think about the potential consequences of some procedures. If the same case were strictly handled adhering to soft tissue sarcoma protocols, the patient would have undergone a wide resection and consequently left with considerable hand dysfunction due to the sacrifice of the midforearm-level ulnar and median nerves as well as vessel and soft tissue. As outlined earlier, ideally the surgeon should not see the tumor, with at least a $1-\mathrm{cm}$ resection margin ( $\mathrm{R} 0$ situation). This creates an extensive soft tissue defect at the forearm level that requires flap coverage. Of course, one could argue that in the case of a malignant tumor, the potential survival rate of the pa- tient would have been drastically reduced because of the highly potentiated risk of tumor spread. In terms of sarcoma surgery, the chosen approach would be deemed inappropriate.

\section{Repeated Surgery: Benign Tumor}

Case 5

History and Examination. This 43-year-old man presented with a painful, firm nodule at his left thenar eminence, at times having a lancinating character, especially when he was grasping items. Subjectively, there were no sensorimotor deficits. It was thought that he harbored a cystic lesion, and surgical removal was attempted at another institution. Intraoperatively, a PNST was diagnosed, and the procedure ended with the release of the flexor retinaculum. The patient was referred for tumor removal. On examination, there was a distinct local tenderness, but no Hoffmann-Tinel sign. There was paresthesia on the ulnar side of his thumb. In comparison with the contralateral hand, there was very mild weakness in the abductor pollicis brevis muscle and the opponens (LSUMC Grade 4). There was no atrophy, but a marked hyperesthesia on the volar, radial side of the hand and along the thumb and middle finger. Preoperative MR imaging demonstrated a $1.2-\mathrm{cm}$ lesion close to abductor pollicis brevis muscle and the flexor retinaculum of the hand. After a repeated retinaculum release, a tumor intermingled within some soft tissue scarring was seen (Fig. 6A and $\mathrm{B}$ ). With dissection from the proximal healthy main inleading median nerve branch, a PNST was identified within the radial side branch of the main median nerve trunk. This nerve branched again into the sensory supply between the thumb and index finger and the motor thenar branch. Under microscopic view, an interfascicular plane was entered, and a pseudocapsule as well as an entering (Fig. 6C) and exiting branch (Fig. 6D) could be identified, thus suggesting a schwannoma. The tumor was resected after prior stimulation response monitoring. Results of histopathological studies confirmed the diagnosis.

\section{Reconstruction After Inappropriate Tumor Removal}

Among most larger series of nerve reconstruction due to iatrogenic injury can be found cases of a benign nerve tumor completely resected together with the parent nerve, al- 


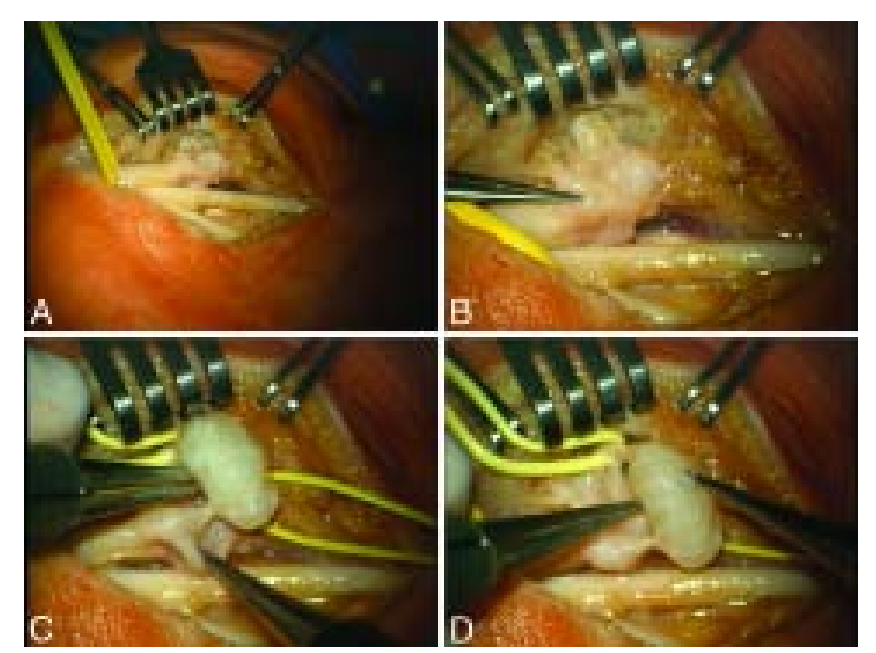

FIG. 6. Case 5. Photographs obtained during repeated surgery for a small but disturbing schwannoma on the left hand median nerve. A: The tumor in relation to the main median nerve branches. The thenar side is retracted, and there is some scar tissue around the tumor. B: Magnified view of the tumor before the dissection was started. C: After microsurgical dissection, a single fascicle leading into the tumor was resected $3 \mathrm{~mm}$ ahead of the tumor. D: A single fascicle leading out of the tumor was also identified and resected $3 \mathrm{~mm}$ past the tumor.

though they are rare. ${ }^{26,27,30-32}$ Figure 7 shows the result of such a resection of the combined tumor and parent nerve: a 4-cm portion of the common peroneal nerve at the knee and above was eradicated. As can be seen from the stump configuration, this procedure must have been performed in a rather blunt fashion. In our own series of 191 iatrogenic nerve injuries surgically treated between January 1990 and December 2002, we identified five cases involving the removal of a BPNST together with the parent nerve and grafting (peroneal nerve, two cases; tibial nerve, one case; femoral nerve, one case; and brachial plexus, one case).

Reconstruction should be attempted in cases in which schwannoma and neurofibroma have been resected along with the parent nerve, leaving the patient with a functional deficit. In such cases we would apply the same principles as those used for other traumatic nerve lesions, given that no malignancy was involved. . $^{2730}$

One of the most important issues is timing. ${ }^{29}$ Ideally, patients who need reconstruction should and can undergo grafting as soon as possible. If there is no doubt about the nature of the lesion, then there is no indication for monitoring alone. Better results will be achieved if reconstruction is performed no later than 6 months after lesioning. ${ }^{23} \mathrm{~A}$ delay will only diminish the potential for functional, useful recovery in motor nerves. If there are concerns about additional blunt injuries, maximum extension of the stump neuromas should be awaited, which will usually be reached by 3 weeks after lesioning. In practice, however, such cases might be presented rather late as opposed to early. At times, it can be difficult to obtain proper information regarding the nature of the lesion. Was the whole nerve originally resected, or was the nerve damaged during tumor manipulation/ exenteration but morphologically left grossly intact? The pathology report should be available in each of these cases to confirm the tumor type and grade and to provide further

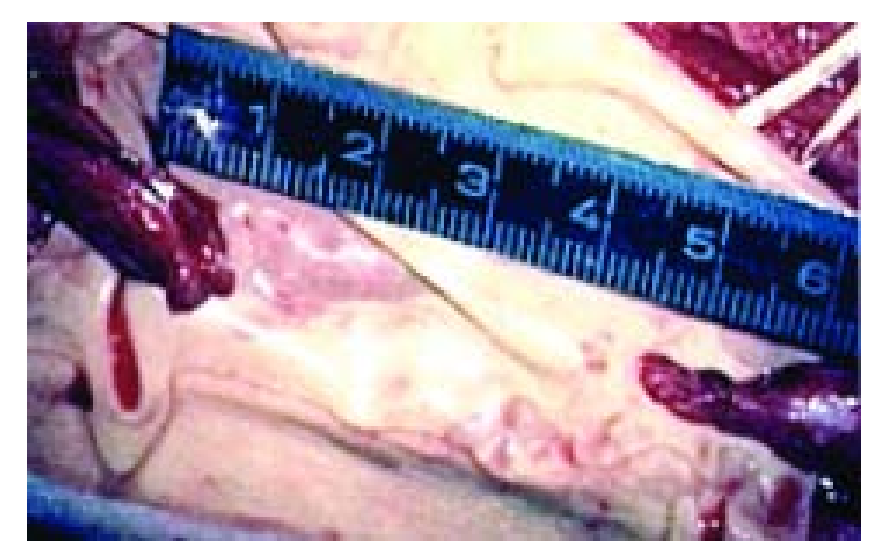

FIG. 7. Photograph illustrating common peroneal nerve stumps after a benign schwannoma was completely resected with the parent nerve.

clues as to the removal of normal nerve tissue..$^{30}$

In contrast, grafts are not an option after resection of MPNSTs for three major reasons: tumor spread, graft failure, and radiotherapy (which prevents the graft from taking). Apart from those considerations, the same principles as those for any other nerve reconstruction should apply; the approaches are nerve dependent and identical to those chosen for other traumatic nerve injuries. ${ }^{28,30}$ Certainly it is very important to approach the pathological entity from distal and proximal healthy tissue to reach correct planes for dissection, to localize the stumps without digging holes in scar tissue, and to prevent further damage of healthy nerve. The sural nerve is usually used for grafts. It is freed from soft tissue, cut to the appropriate length using a razor-sharp blade, and microsurgically coadapted in a epi- or interfascicular manner, ideally covering the whole caliber of the nerve stumps. Care is taken to ensure a tensionless interposition of the graft. The distal and proximal stumps must be resected back to healthy tumor-free fascicular tissue. To rule out a bluntly damaged stretch of stump, the fascicles should be seen to emerge and protrude at the freshly cut plane. Somewhat different from the usual trauma approach is the occasional use of quick section, which is helpful to confirm a tumor- and/or neuroma-free fascicular pattern both proximal and distal to the stumps. In contrast to the diagnostic dilemma of distinguishing benign from malignant features with a quick section, it should be no problem to discern fascicular from tumorous tissue with thin axial slices from the stumps. In patients with von Recklinghausen disease, the sural nerve could potentially contain nerve tumors as well. It is thus prudent to check the legs by palpation preoperatively for potential sural nerve tumors. Although there was no such case in our limited series thus far, a split graft repair may be indicated if a portion of the nerve is left intact during exploration and the rest of the circumference was excised. As for prognosis after reconstruction, it is difficult to quote any evidence-based data because there are none. Only a few nerve reconstruction or nerve tumor studies have been focused on this subject let alone detailed ones. Theoretically, there is the potential risk of new growth at the reconstruction site. Given that the benign tumor mass has been completely removed, however, the same conditions as those for other nerve injuries should apply; therefore, the 
time interval since injury, patient age, level of affected nerve (proximal compared with distal), affected nerve, and sometimes the length of the gap that must be bridged (for example, peroneal nerve) should influence prognosis. Do patients with neurofibromatosis have a higher risk of tumor recurrence at the graft site? We cannot answer this question from experience or evidence.

The aforementioned reconstructive regimen does not apply to plexiform neurofibromas. Although not yet malignant, these lesions are the exception; graft repair after resection of these lesions is controversial. It is the nature of the plexiform neurofibroma to involve the parent nerve, not only along its whole circumference but also along its length. Plexiform neurofibromas are regarded as potential precursors of malignancy and have a 5\% risk of such transformation. Generally, there is a $15 \%$ risk of malignant transformation of a neurofibroma in patients with neurofibromatosis Type 1 , and thus the propensity to transform from a plexiform neurofibroma to an MPNST might be even higher. To our knowledge, the cases of graft repair following plexiform neurofibroma resection in the literature were not characterized by useful functional recovery.

\section{Conclusions}

Peripheral nerve tumor surgery is not always as straightforward as the standard principles and techniques of removal might imply, especially in cases of MPNSTs. Given the absence of better data, it is at times impossible to decide, based on evidentiary reasoning, which form of MPNST resection might be the most beneficial for an individual. Even with supposedly good indicators of pathological type, it is only prudent to be prepared for unexpected findings. Therefore, it is advisable to anticipate different scenarios and to devise different management plans. The possible scenarios and their different effects on functionality, prognosis, and further therapy must be discussed with the patient preoperatively. We believe that damaged nerves following benign tumor removal must be treated like traumatic injuries and thus need repair.

\section{References}

1. Angelov L, Davis A, O'Sullivan B, Bell R, Guha A: Neurogenic sarcomas: experience at the University of Toronto. Neurosurgery 43:56-65, 1998

2. Angelov L, Guha A: Peripheral nerve tumors, in Bernstein M, Berger M (eds): Neuro-Oncology: The Essentials. New York: Thieme, 2000, pp 434-444

3. Baehring JM, Betensky RA, Batchelor TT: Malignant peripheral nerve sheath tumor: the clinical spectrum and outcome of treatment. Neurology 61:696-698, 2003

4. Bhargava R, Parham DM, Lasater OE, Chari RS, Chen G, Fletcher BD: MR imaging differentiation of benign and malignant peripheral nerve sheath tumors: use of the target sign. Pediatr Radiol 27:124-129, 1997

5. Brenner W, Friedrich RE, Gawad KA, Hagel C, von Deimling A, de Wit M, et al: Prognostic relevance of FDG PET in patients with neurofibromatosis type-1 and malignant peripheral nerve sheath tumors. Eur J Nucl Med Mol Imaging 33:428-432, 2006

6. Cardona S, Schwarzbach M, Hinz U, Dimitrakopoulou-Strauss A, Attigah N, Mechtersheimer section sign G, et al: Evaluation of F18-deoxyglucose positron emission tomography (FDG-PET) to assess the nature of neurogenic tumors. Eur J Surg Oncol 29: 536-541, 2003
7. Cheng EY, Dusenbery KE, Winters MR, Thompson RC: Soft tissue sarcomas: preoperative versus postoperative radiotherapy. J Surg Oncol 61:90-99, 1996

8. Cheng EY, Springfield DS, Mankin HJ: Frequent incidence of extrapulmonary sites of initial metastasis in patients with liposarcoma. Cancer 75:1120-1127, 1995

9. Coindre JM, Trojani M, Contesso G, David M, Rouesse J, Bui $\mathrm{NB}$, et al: Reproducibility of a histopathologic grading system for adult soft tissue sarcoma. Cancer 58:306-309, 1986

10. Cormier JN, Pollock RE: Soft tissue sarcomas. Ca Cancer J Clin 54:94-109, 2004

11. Donner TR, Voorhies RM, Kline DG: Neural sheath tumors of major nerves. J Neurosurg 81:362-373, 1994

12. Ducatman BS, Scheithauer BW, Piepgras DG, Reiman HM, Ilstrup DM: Malignant peripheral nerve sheath tumors. A clinicopathologic study of 120 cases. Cancer 57:2006-2021, 1986

13. Dupuy DE, Rosenberg AE, Punyaratabandhu T, Tan MH, Mankin HJ: Accuracy of CT-guided needle biopsy of musculoskeletal neoplasms. AJR Am J Roentgenol 171:759-762, 1998

14. Eilber FR, Eckardt J: Surgical management of soft tissue sarcomas. Semin Oncol 24:526-533, 1997

15. Ferner RE, Gutmann DH: International consensus statement on malignant peripheral nerve sheath tumors in neurofibromatosis. Cancer Res 62:1573-1577, 2002

16. Ferner RE, Lucas JD, O'Doherty MJ, Hughes RA, Smith MA, Cronin BF, et al: Evaluation of (18)fluorodeoxyglucose positron emission tomography ((18)FDG PET) in the detection of malignant peripheral nerve sheath tumors arising from within plexiform neurofibromas in neurofibromatosis 1 . J Neurol Neurosurg Psychiatry 68:353-357, 2000

17. Fleming JB, Cantor SB, Varma DG, Holst D, Feig BW, Hunt $\mathrm{KK}$, et al: Utility of chest computed tomography for staging in patients with T1 extremity soft tissue sarcomas. Cancer 92: 863-868, 2001

18. Ganju A, Roosen N, Kline DG, Tiel RL: Outcomes in a consecutive series of 111 surgically treated plexal tumors: a review of the experience at the Louisiana State University Health Sciences Center. J Neurosurg 95:51-60, 2001

19. Hagel C, Zils U, Peiper M, Kluwe L, Gotthard S, Friedrich RE, et al: Histopathology and clinical outcome of NF1-associated vs. sporadic malignant peripheral nerve sheath tumors. J Neurooncol 82:187-192, 2007

20. Hanna SL, Fletcher BD: MR imaging of malignant soft-tissue tumors. Magn Reson Imaging Clin N Am 3:629-650, 1995

21. Heslin MJ, Smith JK: Imaging of soft tissue sarcomas. Surg Oncol Clin N Am 8:91-107, 1999

22. Hsu CH, Lee CM, Wang FC, Fang CL: Neurofibroma with increased uptake of [F-18]-fluoro-2 deoxy-D-glucose interpreted as a metastatic lesion. Ann Nucl Med 17:609-611, 2003

23. Kandenwein JA, Kretschmer T, Engelhardt M, Richter HP, Antoniadis G: Surgical interventions for traumatic lesions of the brachial plexus: a retrospective study of 134 cases. J Neurosurg 103:614-621, 2005

24. Karakousis CP, Driscoll DL: Treatment and local control of primary extremity soft tissue sarcomas. J Surg Oncol 71:155-161, 1999

25. Kattan MW, Leung DH, Brennan MF: Postoperative nomogram for 12-year sarcoma-specific death. J Clin Oncol 20:791-796, 2002

26. Khan R, Birch R: Iatropathic injuries of peripheral nerves. J Bone Joint Surg Br 83:1145-1148, 2001

27. Kim DH, Murovic JA, Tiel RL, Moes G, Kline DG: A series of 397 peripheral neural sheath tumors: 30 -year experience at Louisiana State University Health Sciences Center. J Neurosurg 102:246-255, 2005

28. Kim DH, Ryu S, Tiel RL, Kline DG: Surgical management and results of 135 tibial nerve lesions at the Louisiana State University Health Sciences Center. Neurosurgery 53:1114-1125, 2003

29. Kline DG, Hackett ER: Reappraisal of timing for exploration of 
civilian peripheral nerve injuries. Surgery 78:54-65, 1975

30. Kline DG, Hudson AR: Nerve Injuries: Operative Results for Major Nerve Injuries, Entrapments, and Tumors. Philadelphia: WB Saunders, 1995

31. Kretschmer T, Antoniadis G, Borm W, Richter HP: [Iatrogenic nerve injuries. Part 1: frequency distribution, new aspects, and timing of microsurgical treatment.] Chirurg 75:1104-1112, 2004 (Ger)

32. Kretschmer T, Antoniadis G, Braun V, Rath SA, Richter HP: Evaluation of iatrogenic lesions in 722 surgically treated cases of peripheral nerve trauma. J Neurosurg 94:905-912, 2001

33. Levine E, Huntrakoon M, Wetzel LH: Malignant nerve-sheath neoplasms in neurofibromatosis: distinction from benign tumors by using imaging techniques. AJR Am J Roentgenol 149: 1059-1064, 1987

34. Murphey MD, Smith WS, Smith SE, Kransdorf MJ, Temple HT: From the archives of the AFIP. Imaging of musculoskeletal neurogenic tumors: radiologic-pathologic correlation. Radiographics 19:1253-1280, 1999

35. Nieweg OE, Pruim J, van Ginkel RJ, Hoekstra HJ, Paans AM, Molenaar WM, et al: Fluorine-18-fluorodeoxyglucose PET imaging of soft-tissue sarcoma. J Nucl Med 37:257-261, 1996

36. O'Sullivan B, Davis AM, Turcotte R, Bell R, Catton C, Chabot $\mathrm{P}$, et al: Preoperative versus postoperative radiotherapy in softtissue sarcoma of the limbs: a randomised trial. Lancet 359: 2235-2241, 2002

37. Perrin RG, Guha A: Malignant peripheral nerve sheath tumors. Neurosurg Clin N Am 15:203-216, 2004

38. Pilavaki M, Chourmouzi D, Kiziridou A, Skordalaki A, Zarampoukas T, Drevelengas A: Imaging of peripheral nerve sheath tumors with pathologic correlation: pictorial review. Eur J Radiol 52:229-239, 2004

39. Russell WO, Cohen J, Enzinger F, Hajdu SI, Heise H, Martin RG, et al: A clinical and pathological staging system for soft tissue sarcomas. Cancer 40:1562-1570, 1977

40. Sadoski C, Suit HD, Rosenberg A, Mankin H, Efird J: Preoperative radiation, surgical margins, and local control of extrem- ity sarcomas of soft tissues. J Surg Oncol 52:223-230, 1993

41. Schulte M, Brecht-Krauss D, Werner M, Hartwig E, Sarkar MR, Keppler P, et al: Evaluation of neoadjuvant therapy response of osteogenic sarcoma using FDG PET. J Nucl Med 40:1637-1643, 1999

42. Schulte M, Mutschler W, Bombelli M: [The value of ultrasound for correction of clinical diagnostic errors in soft tissue tumors.] Bildgebung 61:65-71, 1994 (Ger)

43. Solomon SB, Semih Dogan A, Nicol TL, Campbell JN, Pomper MG: Positron emission tomography in the detection and management of sarcomatous transformation in neurofibromatosis. Clin Nucl Med 26:525-528, 2001

44. Stull MA, Moser RP Jr, Kransdorf MJ, Bogumill GP, Nelson MC: Magnetic resonance appearance of peripheral nerve sheath tumors. Skeletal Radiol 20:9-14, 1991

45. Varma DG, Moulopoulos A, Sara AS, Leeds N, Kumar R, Kim EE, et al: MR imaging of extracranial nerve sheath tumors. J Comput Assist Tomogr 16:448-453, 1992

46. Woodruff JM, Godwin TA, Erlandson RA, Susin M, Martini N: Cellular schwannoma: a variety of schwannoma sometimes mistaken for a malignant tumor. Am J Surg Pathol 5:733-744, 1981

47. Wunder JS, Healey JH, Davis AM, Brennan MF: A comparison of staging systems for localized extremity soft tissue sarcoma. Cancer 88:2721-2730, 2000

48. Zhou H, Coffin CM, Perkins SL, Tripp SR, Liew M, Viskochil DH: Malignant peripheral nerve sheath tumor: a comparison of grade, immunophenotype, and cell cycle/growth activation marker expression in sporadic and neurofibromatosis 1-related lesions. Am J Surg Pathol 27:1337-1345, 2003

Manuscript submitted March 20, 2007.

Accepted April 26, 2007.

Address reprint requests to: Thomas Kretschmer, M.D., Ph.D., Neurochirurgische Klinik der Universität Ulm, Ludwig-HeilmeyerStrasse 2,89312 Günzburg, Germany. email: thomas.kretschmer@ uni-ulm.de. 